Supplementary Information

\title{
Medicated Janus fibers fabricated using a Teflon-coated side-by-side spinneret
}

Deng-Guang Yu ${ }^{\mathrm{a}, *}$, Chen Yang ${ }^{\mathrm{a}}$, Miao Jin ${ }^{\mathrm{b}}$, Gareth R. Williams ${ }^{\mathrm{b}}$, Hua Zou ${ }^{\mathrm{a}}$, Xia Wang ${ }^{\mathrm{a}, * *}$, SW Annie Bligh ${ }^{\mathrm{c}, * * *}$

\footnotetext{
${ }^{a}$ School of Materials Science \& Engineering, University of Shanghai for Science and Technology, Shanghai 200093, China.

${ }^{\mathrm{b}}$ UCL School of Pharmacy, University College London, London WC1N 1AX, UK.

${ }^{\mathrm{c}}$ Faculty of Science and Technology, University of Westminster, 115 New Cavendish Street, London W1W 6UW, UK.
}

\section{* Corresponding authors:}

E-mail: *ydg017@usst.edu.cn (DG Yu); **wangxia@usst.edu.cn (X Wang); ***a.bligh@westminster.ac.uk (SWA Bligh) 


\section{Experimental}

Differential scanning calorimetry (DSC) was conducted using an MDSC 2910 differential scanning calorimeter (TA Instruments Co., USA). Sealed samples were heated at $5{ }^{\circ} \mathrm{C} / \mathrm{min}$ from ambient temperature to $200{ }^{\circ} \mathrm{C}$ under a flow of nitrogen gas (40 $\mathrm{mL} / \mathrm{min}$ ). X-ray diffraction (XRD) was carried out with a D/Max-BR diffractometer (RigaKu, Japan) with $\mathrm{Cu} \mathrm{K} \alpha$ radiation over the $2 \theta$ range $5^{\circ}$ to $60^{\circ}$ at 40 $\mathrm{kV}$ and $30 \mathrm{~mA}$. Attenuated total reflectance-Fourier transform infrared (ATR-FTIR) spectra were recorded on a Nicolet-Nexus 670 FTIR spectrometer (Nicolet Instrument Corporation, USA) over the range $4000 \mathrm{~cm}^{-1}$ to $500 \mathrm{~cm}^{-1}$ at a resolution of $2 \mathrm{~cm}^{-1}$.

\section{The side-by-side electrospinning processes}

A diagram of the side-by-side electrospinning process is shown in Fig. S1a. Two working fluids are separately driven by two syringe pumps through a side-by-side spinneret. Under an appropriate applied voltage, the two fluids jointly form a compound Janus Taylor cone. Janus fibers are collected on a grounded collector, randomly arranged in a non-woven mat.
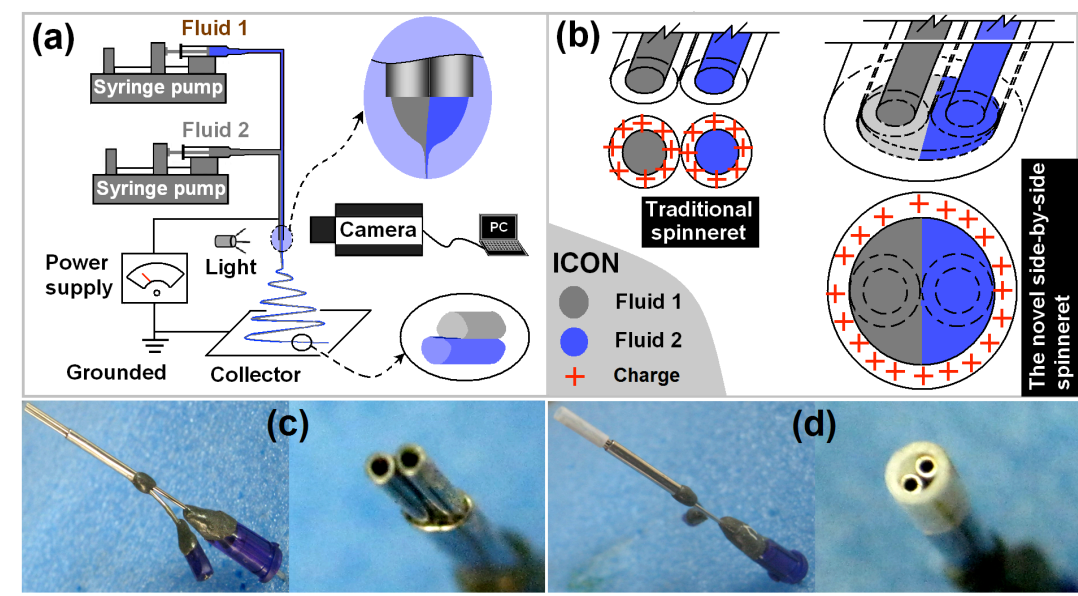

Fig. S1. The side-by-side electrospinning process and spinnerets: (a) the experimental apparatus; (b) schematic diagrams of the traditional side-by-side and the Teflon-coated spinnerets; photographs of (c) the traditional side-by-side spinneret; and (d) the Teflon-coated spinneret. 
Fig. S1b depicts diagrams of the traditional side-by-side spinneret and the novel spinneret developed in this study. Photographs are exhibited in Fig. S1c and S1d. The new spinneret is simply prepared by coating the two parallel metal capillaries with a section of Teflon tube on their exterior, ensuring it extends slightly beyond their termini.

Physical form and component compatibility Differential scanning calorimetry data are given in Fig. S2. The raw ketoprofen (KET) material exhibits a single sharp endothermic response corresponding to melting at $95.7{ }^{\circ} \mathrm{C}\left(\Delta H_{f}=-115 \mathrm{~J} / \mathrm{g}\right)$, demonstrating that the pure drug exists as a crystalline material. Polyvinylpyrrolidone (PVP) K60, PVP K10 and ethyl cellulose (EC) do not show any fusion peaks or phase transitions, verifying that they are amorphous polymers. All the fibers, both monolithic and Janus, have similar DSC traces, with no KET melting endotherm present for any of the systems. These findings indicate that KET was converted into the amorphous physical form by dint of the electrospinning process.

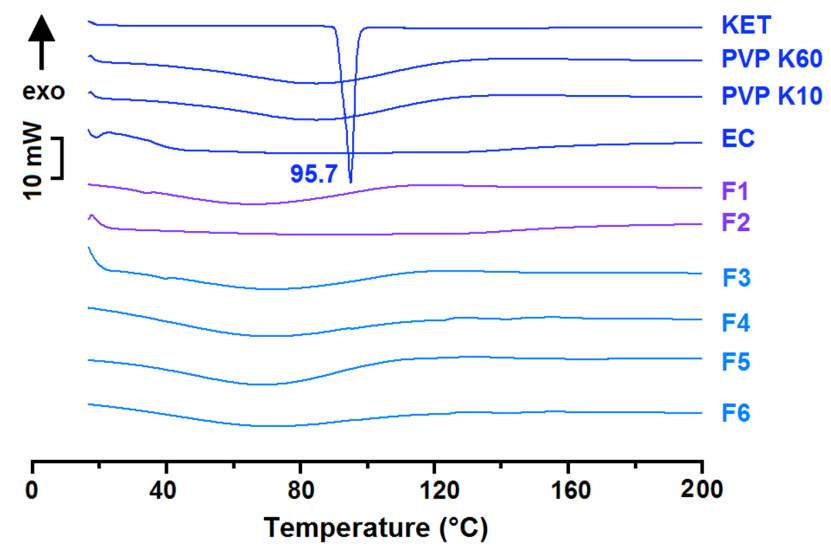

Fig. S2. DSC thermograms of the raw materials and fibers F1 to F6.

XRD patterns of the raw materials and their fibers are shown in Fig. S3. The raw KET material is clearly crystalline, and its pattern contains many distinct Bragg 
reflections. The patterns of the polymers show a diffuse background pattern with double diffraction halos, indicating all the polymers are amorphous. In the patterns of the fibers F1 to F6 no reflections from KET are found, confirming that KET was no longer present as a crystalline material, but was transformed into an amorphous composite during the electrospinning processes. The XRD results thus concur with the DSC data. These results also agree with the FESEM and TEM observations, in which no particles resulting from phase separation can be discerned.

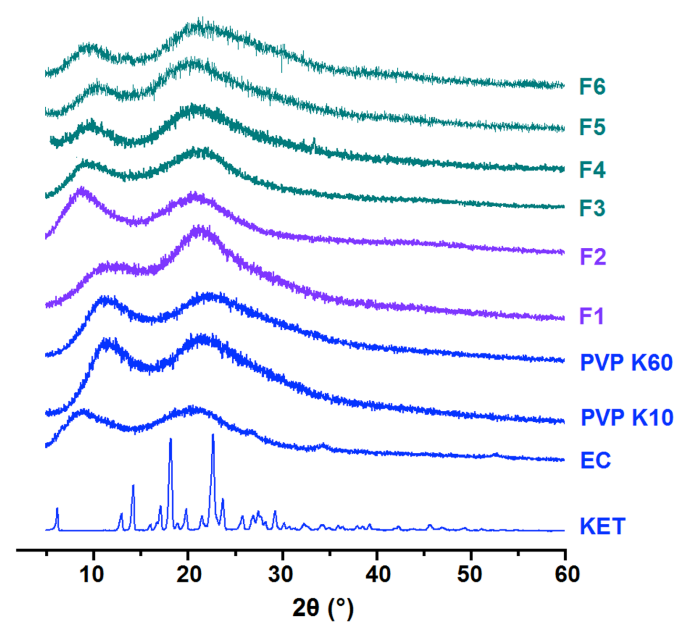

Fig. S3. XRD patterns of the raw materials and fibers F1 to F6.

The molecular structures of the raw materials, their infrared (IR) spectra and the spectra of the electrospun fibers are illustrated in Fig. S4. The pure crystalline KET material has two characteristic peaks at $1697 \mathrm{~cm}^{-1}$ and $1658 \mathrm{~cm}^{-1}$. KET has two carbonyl groups and one hydroxyl group, and in its crystalline form pairs of molecules associate in dimers through hydrogen bonding. The IR peak at $1697 \mathrm{~cm}^{-1}$ can be assigned to the stretching vibration of the carbonyl group in the KET dimer, and the peak at $1658 \mathrm{~cm}^{-1}$ is a result of stretching of the ketone group. For all the fibers, the peak at $1697 \mathrm{~cm}^{-1}$ cannot be seen in the IR spectra. 
In addition, almost all the peaks in the fingerprint region of KET have greatly decreased in intensity, or completely disappeared, in the fibers' spectra. The characteristic carbonyl peak at $1661 \mathrm{~cm}^{-1}$ in the PVP spectrum exhibits a slight red shift to $1655-1657 \mathrm{~cm}^{-1}$ in the spectra of all the PVP-containing fibers. Taken together, these observations indicate that KET dimers do not exist in the fibers, and that instead the KET molecules have formed composites with their polymeric carriers. PVP and KET have carbonyl groups to act as potential proton receptors, whereas EC and KET possess free hydroxyl groups which can act as proton donors. Thus hydrogen bonding between KET and PVP should occur in all fibers bar F2, hydrogen bonding between KET and EC should occur in all fibers except F1, and hydrogen bonding between EC and PVP should occur in fibers F4 to F6.
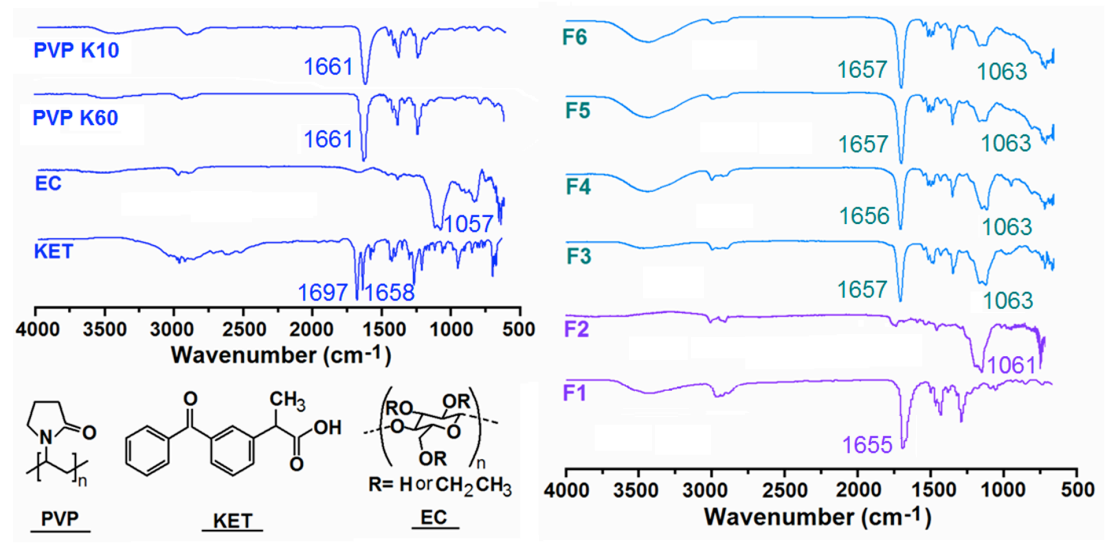

Fig. S4. ATR-FTIR spectra of the raw materials and fibers F1 to F6, together with the molecular structures of KET, PVP and EC.

By interacting with the polymer carriers, KET molecules are less likely to form the dimers that are essential for a crystalline lattice. The existence of hydrogen bonding, combined with possible hydrophobic interactions between the benzene ring of KET and aliphatic groups in the polymers, should ensure that the individual components in the fibers have good compatibility and give the fibers good physical 
stability. In addition, the spectra of the Janus fibers F3 to F6 appear to comprise a superposition of the spectra of F1 and F2, suggesting the presence of two separate nanocomposites in all the Janus structures.

\section{Drug release mechanism}

To investigate the drug release mechanism, the fibers were removed from the dissolution cells after $24 \mathrm{~h}$ of in vitro dissolution tests. These fibers were dried under ambient conditions, after which FESEM observations were carried out. Fig. S5 depicts the remains of fibers F3 to F6. Since the PVP K60 side of the fibers dissolves immediately upon addition to the aqueous medium, the material left after $24 \mathrm{~h}$ will comprise the EC side of the Janus structures. Although the overall width of the initial Janus fibers was not affected by the addition of PVP K10 to the EC side, the EC component remaining after $24 \mathrm{~h}$ appears in general to get smaller with an increase of PVP K10 content. The remains of F3 to F6 exhibit increasingly curved fibers.

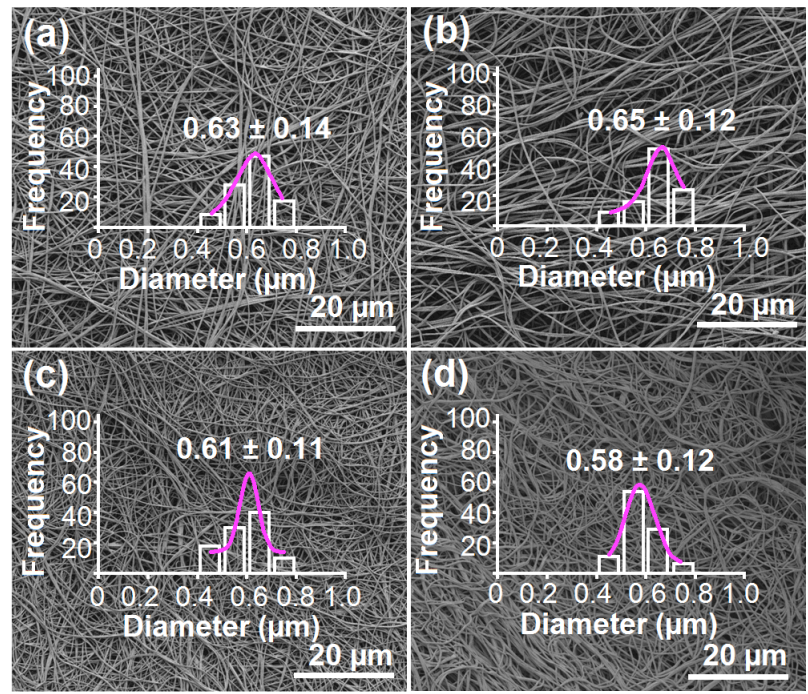

Fig. S5. FESEM images of the fibers remaining after $24 \mathrm{~h}$ of in vitro dissolution tests and their size distributions: (a) F3; (b) F4; (c) F5; and (d) F6. 